\title{
Differential effects of shared attention on perception of heading and 3-D object motion
}

\author{
CONSTANCE S. ROYDEN and ELLEN C. HILDRETH \\ Wellesley College, Wellesley, Massachusetts
}

\begin{abstract}
When a person moves in a straight line through a stationary environment, the images of object surfaces move in a radial pattern away from a single point. This point, known as the focus of expansion (FOE), corresponds to the person's direction of motion. People judge their heading from image motion quite well in this situation. They perform most accurately when they can see the region around the FOE, which contains the most useful information for this task. Furthermore, a large moving object in the scene has no effect on observer heading judgments unless it obscures the FOE. Therefore, observers may obtain the most accurate heading judgments by focusing their attention on the region around the FOE. However, in many situations (e.g., driving), the observer must pay attention to other moving objects in the scene (e.g., cars and pedestrians) to avoid collisions. These objects may be located far from the FOE in the visual field. We tested whether people can accurately judge their heading and the three-dimensional (3-D) motion of objects while paying attention to one or the other task. The results show that differential allocation of attention affects people's ability to judge 3-D object motion much more than it affects their ability to judge heading. This suggests that heading judgments are computed globally, whereas judgments about object motion may require more focused attention.
\end{abstract}

As we move through the world, we often confront numerous stationary and moving obstacles that we must identify and avoid. To avoid these objects successfully, we must be able to judge their three-dimensional (3-D) motion relative to ourselves. Simultaneously, we must accurately judge our own direction of motion. For example, drivers on busy streets must judge their direction of motion and, at the same time, identify stationary and moving objects, such as pedestrians or other cars. To avoid collisions, a driver must also judge the direction of motion of the other cars and pedestrians. A rapidly moving observer (e.g., a driver of a car on a freeway) must quickly and accurately accomplish these judgments of heading and object motion. It is unclear, however, whether the brain performs these tasks in parallel, without requiring focused attention on one task or the other, or whether it accomplishes these tasks in series, performing shifts of attention to compute heading and 3-D object motion separately. Understanding the attentional requirements of heading and object motion perception can help to evaluate models of the neural mechanisms involved in computing these properties. In the present experiments, we examined how differential allocation of attention in dual-task procedures affects the ability to judge heading and 3-D object motion.

When a person moves in a straight line through a stationary environment, the images of object surfaces on the

This research was supported by NSF Grant SBR-930126. The authors thank Jeremy Wolfe and Kathleen O'Craven for helpful comments and discussion. Correspondence should be addressed to C. S. Royden, Department of Computer Science, Wellesley College, Wellesley, MA 02481 (e-mail: croyden@wellesley.edu). retina move in a radial pattern away from a single point, known as the focus of expansion (FOE), which corresponds to the observer's heading (Figure 1). Numerous studies have shown that observers judge their heading well in this situation (Crowell \& Banks, 1993; Crowell, Royden, Banks, Swenson, \& Sekuler, 1990; Cutting, Springer, Braren, \& Johnson, 1992; Rieger \& Toet, 1985; Royden, Banks, \& Crowell, 1992; Royden, Crowell, \& Banks, 1994; van den Berg, 1992; Warren \& Hannon, $1988,1990)$. They perform best when the FOE is visible (Crowell \& Banks, 1993; Warren \& Kurtz, 1992). Crowell and Banks (1996) have shown that the region around the FOE is the most informative region for judging heading. A moving object in the scene has no effect on the accuracy of heading judgments unless it obscures the FOE, in which case the presence of the moving object causes a bias in heading judgments (Royden \& Hildreth, 1996; Warren \& Saunders, 1995; see also, Cutting, Vishton, \& Braren, 1995). This suggests that the FOE has particular importance in judgments of heading. In all of the above studies, however, observers performed only a single heading task and were free to focus their attention wherever needed. Land and Lee (1994) showed that drivers repeatedly return their gaze to the direction in which they are steering their car, which suggests that the most accurate judgments of heading may require focused attention by the driver. If the accurate computation of heading requires focused attention on the region around the FOE, then one would expect that an observer who is paying attention to another task would show a decline in ability to judge heading. On the other hand, if heading computation is a parallel process, performed without the requirement for the additional processing resources provided by 


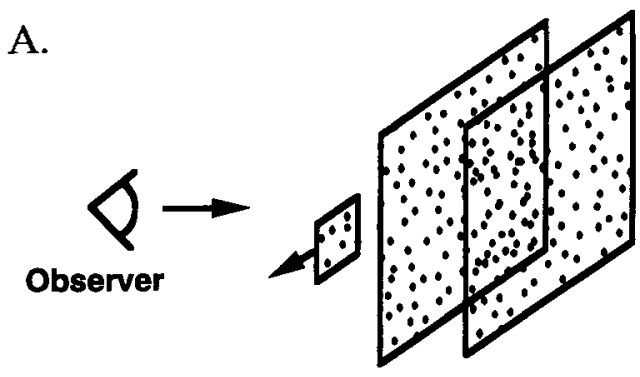

Simulated Scene

B.

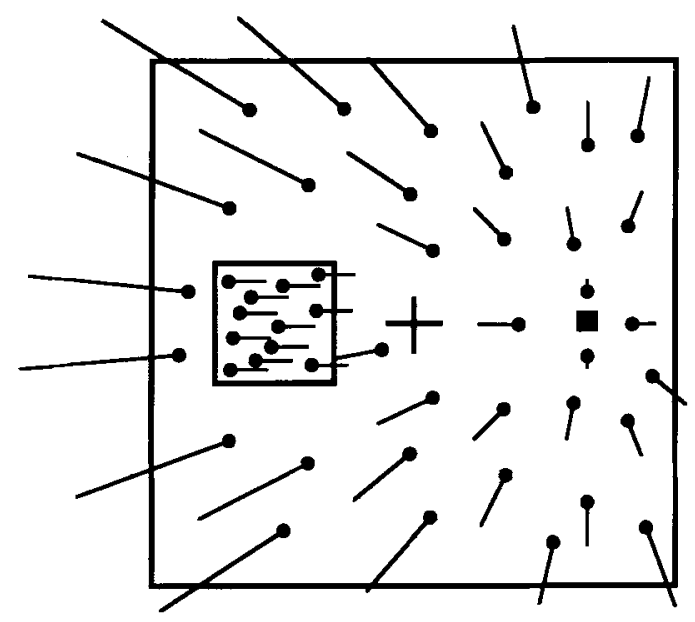

Figure 1. Diagram of simulated observer motion. (A) Schematic showing observer motion toward two transparent frontoparallel planes composed of random dots. An opaque object moves horizontally in front of the planes. (B) Schematic of the optic flow field generated by the observer motion toward the scene diagrammed in $A$. The small black square indicates the position of the focus of expansion (FOE). The cross in the center is the fixation cross that the observers were asked to fixate during each trial. The borders of the moving object are shown for clarity but were not present in the display. The object was defined only by the motion of the dots. Each dot represents a point in the scene, and the line extending outward from the dot is proportional to the speed and direction of the image motion for that dot.

focusing attention on this task, then observers should be able to judge their heading even when allocating their attention elsewhere. The present experiments were carried out to determine which of the above possibilities holds true for human observers.

Many models that compute heading from retinal velocity have difficulty when moving objects are present in the scene, unless the objects can first be identified and discounted from the heading computation. For example, models that use information throughout the scene indiscriminately, such as template models (Hatsopoulos \& Warren, 1991; Perrone \& Stone, 1994) and error minimization models (Bruss \& Horn, 1983; Heeger \& Jepson,
1992), would be adversely affected by the presence of a moving object (see Hildreth \& Royden, 1996, and Royden \& Hildreth, 1996, for a more complete discussion of models). However, models that identify moving objects in a scene often rely on knowledge of the observer's motion parameters. For example, some models use knowledge about the observer's heading to predict the expected flow field pattern and identify moving objects as any group of velocity vectors that are inconsistent with this pattern (see Thompson, Lechleider, \& Stuck, 1993; Thompson \& Pong, 1990). This interrelationship between heading computation and 3-D object detection complicates the judgment of heading in the presence of moving objects. The problem is not insurmountable, however, and several models have been advanced for the computation of heading in the presence of moving objects.

Hildreth (1992) showed how one could accommodate moving objects in the scene by extending a model first presented by Rieger and Lawton (1985), based on the mathematical analysis by Longuet-Higgins and Prazdny (1980). Hildreth's model divides the visual scene into small regions and computes heading estimates for each of these regions simultaneously. The heading that is consistent with the image motion in the majority of these regions is identified as the observer's heading. Regions with motion inconsistent with the identified heading are considered to be moving objects. This computation can easily be done in parallel across the visual image; so, if such a mechanism were used by the visual system, one would not expect the focus of attention to matter in the judgment of heading.

Warren and Saunders (1995) adapted a template model to account for their data on observer motion in the presence of moving objects. Their model uses template cells that recognize the radial flow patterns generated by different observer translational motions. By weighting the influence of velocity information near the FOE more strongly than that of more peripheral regions, the influence of moving objects on heading judgments is reduced, as long as the moving object is sufficiently distant from the FOE. This model can also compute heading in parallel, so one would expect that such a mechanism would not be affected by shifting attention.

A third type of model was suggested by Royden and Hildreth (1996). This model first estimates the observer's heading on the basis of all the velocity information in the scene. This initial estimate can be somewhat biased by the presence of moving objects. The model then revises its heading estimate using only information in the region around the first estimated FOE. This allows fine tuning of the initial estimate, and, if the moving object does not obscure the FOE, the image motion associated with the object will not affect the second heading estimate. Such a model, in which heading is computed by sequential estimates of the location of the FOE, might require the allocation of extra computational resources to that region 
for accurate heading estimates. If so, one would expect that the observer's focus of attention could have a significant effect on heading judgments.

In this study, we examined the effect on heading judgments of requiring the observer to perform an additional task: judgment of 3-D object motion. We investigated the effect of shifting attention by testing how well people perform these two tasks simultaneously, when paying attention only to one or the other.

\section{EXPERIMENT 1 Judging Heading and 3-D Object Motion}

In Experiment 1, we tested whether differential allocation of the observers' attention affects their ability to judge heading and 3-D object motion accurately in a dualtask experiment.

\section{Method}

We used a computer-controlled display of moving random dots to simulate observer motion toward two transparent frontoparallel planes with an opaque object moving in front, as shown in Figure 1. This display was nearly identical to that used in previous experiments (Royden \& Hildreth, 1996). In each trial, lasting $0.4 \mathrm{sec}$, the computer simulated motion toward the two transparent planes at distances of 400 and $1,000 \mathrm{~cm}$ from the observer at the beginning of the trial. The stimulus was presented within a $30^{\circ} \times 30^{\circ}$ window, and dots moving out of the window disappeared from the screen. The two planes consisted of 500 dots at the beginning of each trial; each dot consisted of 1 pixel on the monitor, subtending 3.6 arc min. The stimulus simulated observer motion toward the scene with a speed of $200 \mathrm{~cm} / \mathrm{sec}$ in 1 of 12 directions that deviated from a straight line toward the center of the screen by the following amounts: $4^{\circ}$, $6^{\circ}, 8^{\circ}$, or $10^{\circ}$ horizontally combined with $0^{\circ}, 2^{\circ}$, and $-2^{\circ}$ vertically. All headings were to the right of a central fixation cross, which the observers were asked to fixate during each trial. The opaque object was centered at $10.5^{\circ}$ to the left of center at the start of each trial and moved at $8.1 \% \mathrm{sec}$ horizontally to the right. To eliminate size and density cues, the starting size was randomly selected between $7.6^{\circ}$ and $9.0^{\circ}$ on a side. The density was varied at random between 0.67 and $0.97 \mathrm{dots} / \mathrm{deg}^{2}$. In one condition, the object moved purely horizontally with respect to the observer; its simulated motion in the world was the sum of the observer's simulated velocity plus a horizontal component. Thus, its image on the monitor did not change size during the trial. In the second condition, a component of motion in depth of 1.5 times the additional horizontal component was added to the object motion, so that it came closer to the observer and its image expanded over the course of the trial. These two conditions are shown schematically in Figure 2. The observers viewed the display monocularly at a distance of $30 \mathrm{~cm}$ in a darkened room. The stimuli were generated by an Apple Quadra 950 and presented on an Apple 21 -in. monitor. Stimulus frames were drawn at a rate of $25 \mathrm{~Hz}$. Each condition ( 12 headings $\times 2$ object motions) was presented 10 times for a total of 240 trials per run of the experiment. No feedback was given.

We used two tasks in this experiment. In the heading task, at the end of each trial, the observers were required to move a cursor to the position on the display that corresponded to their perceived heading, which was always simulated to the right of a central fixation point. In the object task, the observers were required to judge whether the moving object, which was in the left half of the stimulus, had a component of motion toward the observer (and thus was expanding) or whether it moved parallel to the image plane of the monitor (and thus remained a constant size). We tested observer responses in four conditions: (1) The observers performed only the heading judgment task; (2) the observers performed only the object motion task; (3) the observers performed both tasks after each trial, but they were instructed to pay attention to the object motion task and do as well as possible on this task; (4) the observers performed both tasks, but they were instructed to pay attention to the heading task and perform as well as possible on that task. The stimuli were identical in all four conditions. The observers were tested on the single-task conditions first, followed by the dual-task conditions. The order of the two conditions within each group (single- or dual-task) was randomized across observers.
A.

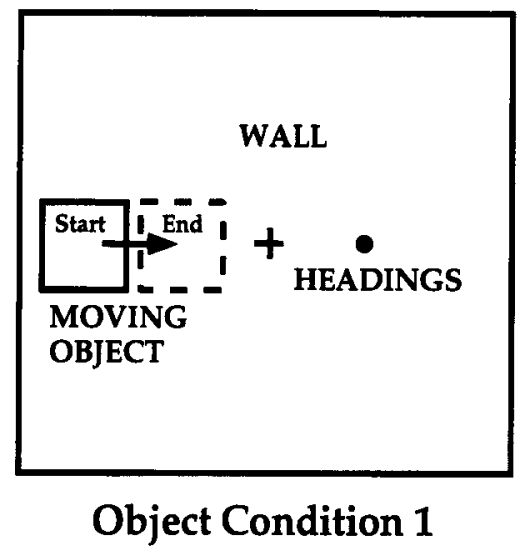

B.

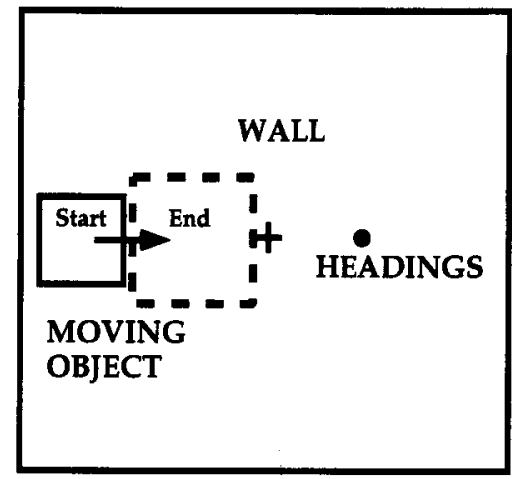

Object Condition 2

Figure 2. Schematic showing the images seen in the two object conditions. (A) Schematic showing the condition in which the object remains a uniform distance from the observer during the trial, and, thus, its image size stays constant. B. Schematic showing the condition in which the object moves toward the observer during the trial, and, thus, its image expands. The cross in the middle of each schematic indicates the fixation cross that the observers fixated during the trial. The dot in the right-hand side of the schematic shows that the simulated headings were always to the right of center; however, the actual heading direction varied within this part of the visual field, as described in the Method section. 
We tested 6 observers in this experiment. Two were experienced psychophysical observers and had knowledge of the hypotheses being tested. The other 4 were volunteers who were paid to participate in this study and were naive to the hypotheses being tested. Three of these were experienced in psychophysical experiments on heading judgments, and the 4th was experienced in other visual psychophysical studies but had not previously participated in studies of heading judgments.

A.

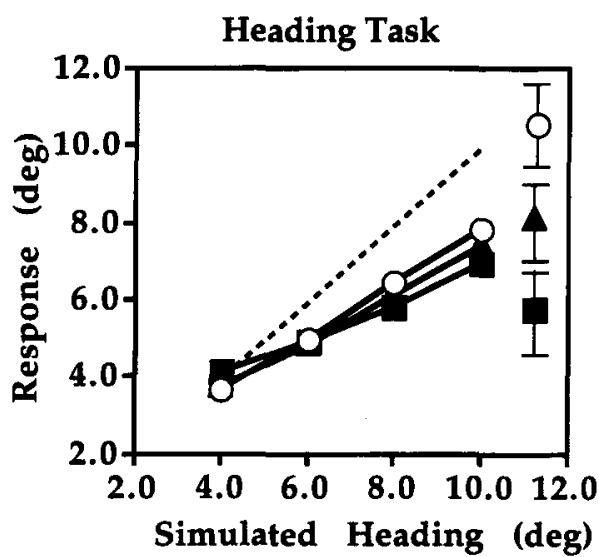

B.

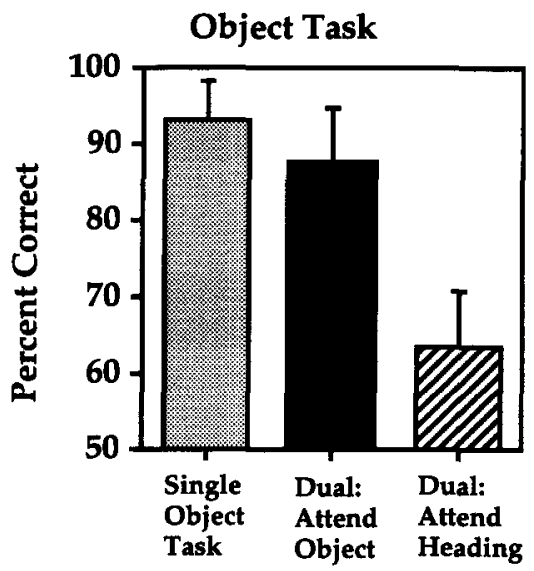

Figure 3. Results of Experiment 1 testing the effects of attention shifts on the observers' ability to judge heading. (A) Average horizontal heading responses for each simulated horizontal heading. Each data point represents the response averaged over 10 trials for each of the three vertical headings and two object conditions (60 trials total) and 6 observers. The dashed line indicates the expected results if the observers chose the exact heading simulated. The open circles indicate responses when the observers performed the heading task alone. The filled symbols show the average heading responses when the observers performed both tasks. Filled squares show responses when the observers attended to the object task. Filled triangles show the average response when the observers attended the heading task. The vertical lines to the right of the graph indicate 2 standard deviations across observers, averaged over the four headings, for each condition. (B) Results for the object task. The shaded bar shows the percent correct for the object task alone. The black bar shows the percent correct when the observers performed both tasks and attended to the object task. The hatched bar shows the results when the observers performed both tasks and attended the heading. Error bars indicate 1 standard deviation across observers.

\section{Results}

The results of Experiment 1 are diagrammed in Figure 3. Because the horizontal heading error was similar for all three vertical headings, the vertical heading data were combined so that only the horizontal headings are shown. Thus, each data point in Figure $3 \mathrm{~A}$ represents the horizontal heading response averaged for 6 observers each performing the task 60 times ( 3 vertical headings $X$ 2 object conditions $\times 10$ trials/condition). The accuracy on the object task did not vary much for the different simulated headings, so the data in Figure 3B show the percent correct averaged for the 6 observers each performing the task 240 times ( 12 heading conditions $\times 2$ object conditions $\times 10$ trials per condition). The observers performed very well on each task when performed alone, achieving a level of $92.6 \%$ correct responses for the object task (shaded bar in Figure 3B) and an average error of $1.3^{\circ}$ in the heading task (open circles in Figure 3A). The observers showed a slight central bias in heading judgments, as has been seen in previous experiments (Cutting et al., 1992; Johnston, White, \& Cumming, 1973; Llewellyn, 1971; Royden \& Hildreth, 1996; Warren \& Saunders, 1995). When performing both tasks, the observers continued to judge heading well both when they paid attention to the heading task (average error $=1.5^{\circ}$; filled triangles in Figure $3 \mathrm{~A}$ ) and when they paid attention to the object task (average error $=1.6^{\circ}$; filled squares in Figure $3 \mathrm{~A}$ ). As can be seen in the graphs, there was little difference in heading responses in both these conditions. While the central bias appeared to be slightly higher in the dual-task conditions, a two-way analysis of variance (ANOVA), using multivariate repeated measures design with a Greenhouse-Geisser correction (Geisser \& Greenhouse, 1958), comparing all of the conditions for which there was a heading task showed that the difference was not significant $[F(2,60)=0.448, p>.5]$.

In contrast, in the dual-task conditions, the observers performed well on the object task only when paying attention to this task, responding correctly on $87.2 \%$ of the trials in this condition (solid bar in Figure 3B). When paying attention to the heading task, the observers' accuracy on the object task fell dramatically to $63.4 \%$ correct (hatched bar in Figure 3B). An ANOVA of these results showed a significant effect of attention $[F(2,15)=26.5$, $p<.0005]$. Planned comparisons showed a significant difference between the two dual-task conditions [attend object vs. attend heading, $F(1,15)=31.06, p<.001]$ but no significant difference between the single- and dualtask (attend-object) conditions $[F(1,15)=1.616, p>.2]$. The variation in results for the object task in the different attention conditions indicates that the observers shifted their attention when instructed to do so, and they were unable to perform the object task well when not paying attention to it. This suggests that the judgment of object motion is a "resource-limited" task - that is, it is sensitive to the amount of resources allocated to the task (Norman \& Bobrow, 1975). On the other hand, the results for the heading task indicate that the visual system calculates 
heading accurately even when the observer is not directly attending to that task. This task can be described as "datalimited" (Norman \& Bobrow, 1975).

\section{EXPERIMENT 2 Limited Viewing Window for Heading}

The results of Experiment 1 are surprising. Because both tasks involve judgments of the relative motion between the observer and a surface, one might expect that performance on the two tasks would deteriorate symmetrically when attention is shifted away from each. One difference between the heading task and the moving object task in Experiment 1 is the spatial extent of the visual information that observers can use to solve the task. The information for the heading task fills a window that is $30^{\circ} \times 30^{\circ}$ and is present everywhere except in the region containing the moving object. Consequently, there is much information about heading in both the left half and the right half of the visual field. However, information about the object task is limited to the small region (about $8^{\circ} \times 8^{\circ}$ ) in the left half of the visual field. Conceivably, when we instruct observers to attend to a particular task, they may localize their attention spatially. Thus, they might attend to the left half of the visual field when attending to the object task and attend to the right half of the visual field when attending to the heading task. Because there is information about heading in the left half of the visual field, observers might make use of this information to compute heading while attending to the object task. There is no information about the object in the right half of the visual field, so observers could not use the same strategy to compute the object motion while attending to the heading task. In Experiment 2, we examined this possibility by removing the heading information in the left half of the visual field.

\section{Method}

Experiment 2 was identical to Experiment 1 except that the dots associated with the heading task were confined to the right half of the visual field. The window for the heading scene was therefore $15^{\circ}$ wide and $30^{\circ}$ high, extending from the center of the screen to the right, as shown in Figure 4. The number of dots in the heading scene was reduced by a factor of 2 , so that the dot density remained the same as that for Experiment 1. The object was present in the left half of the visual field and moved across a blank screen. The tasks were the same as in Experiment 1. Five subjects from Experiment 1 participated in Experiment 2.

\section{Results}

Figure 5 shows the results of Experiment 2. The observers performed the heading task alone very well (average error $=0.4^{\circ}$ ). The horizontal heading data have been averaged over the three vertical headings as in Experiment 1 . There was a slight decrease in heading accuracy when the observers performed two tasks relative to when they performed the heading task alone. However, there was essentially no difference in heading judgments when the observers shifted their attention from the head-

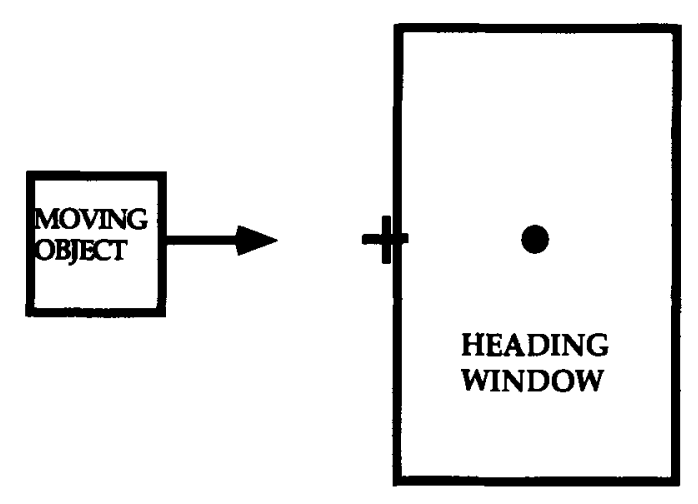

Figure 4. Schematic of image presented during Experiment 2. The rectangle on the right indicates the region in which heading information was presented. Dots representing the two transparent planes toward which the observers were moving appeared only in this region, and they disappeared from the screen when they moved out of this area. The moving object on the left moved across a blank area of the screen. A fixation cross was present in the center of the screen, as before.

ing task (average error $=0.90^{\circ}$ ) to the object task (average error $=1.1^{\circ}$ ). An ANOVA, using repeated measures with the Greenhouse-Geisser correction, showed a nearly significant effect of condition for the three different tasks $[F(2,48)=4.49, p<.052]$. Planned comparisons showed a significant difference between the single- and dual-task (attend-heading) conditions $[F(1,48)=6.012, p<.05]$. There was no significant difference between the two dualtask conditions $[F(1,48)=0.070, p>.7]$.

For the object task alone, the observers were very accurate $(96.8 \%$ correct). When performing both tasks and attending the object, the observers' accuracy fell slightly (to $89 \%$ correct). As in Experiment 1, allocation of attention to the heading task caused a marked decrease in accuracy (to $74.5 \%$ correct). An ANOVA showed a significant effect of attention condition $[F(2,12)=12.64$, $p<.03]$. Planned comparisons showed a significant difference between the two dual-task conditions $[F(1,48)=$ $10.35, p<.03]$. There was no significant difference between the single-task condition and the dual-task condition in which the observers attended the object task ( $p>$ .1). So, as in Experiment 1, different allocation of attention affected judgments of object motion more dramatically than it affected judgments of heading.

\section{EXPERIMENT 3 \\ Heading Window Limited to an $8^{\circ} \times 8^{\circ}$ Square}

While Experiment 2 eliminated the possibility that the differences seen for the heading task and the object task were due to a spatial localization of attention, the heading task in Experiment 2 still had more spatially extended visual information in the stimulus than the object task. The heading task had a large viewing window $\left(15^{\circ}\right.$ $\times 30^{\circ}$ ), containing 250 moving points. The moving ob- 
A.

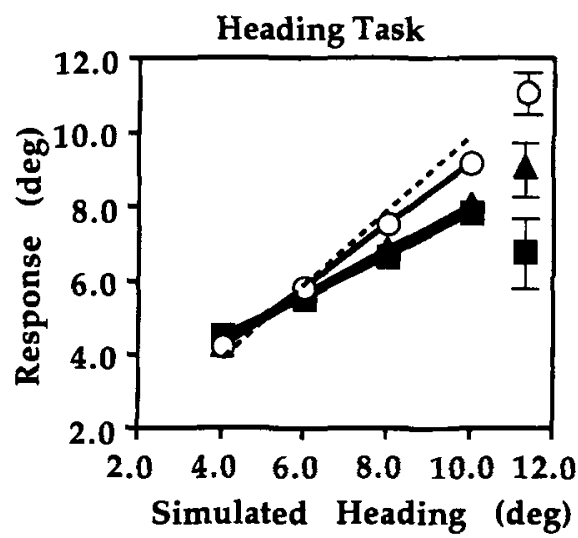

B.

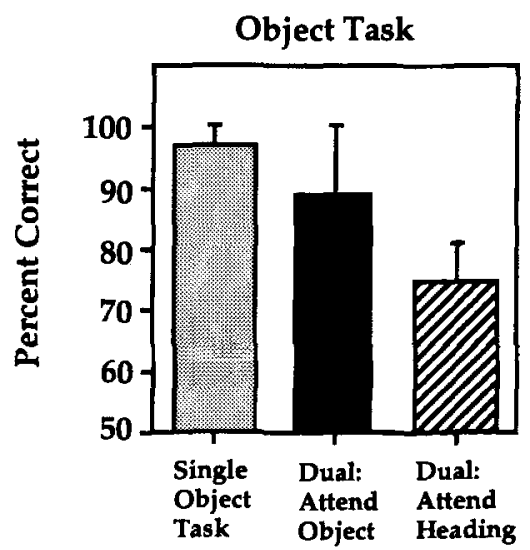

Figure 5. Results of Experiment 2, in which heading information was limited to the right half of the visual field. (A) Results of the heading task for the three heading conditions. (B) Results for the object task. All symbols are as described in Figure 3.

ject, on the other hand, was a square subtending between $7.6^{\circ}$ and $9.0^{\circ}$ on a side, with between 39 and 78 dots. The larger number of dots and larger viewing window provides more information about the heading task than the moving-object task. This difference could lead to the differing attentional effects in the two tasks. To examine this possibility, we reduced the heading window to an $8^{\circ}$ $\times 8^{\circ}$ square.

\section{Method}

Experiment 3 was identical to Experiment 1 except that the size of the clipping window for the heading task was reduced to an $8.0^{\circ} \times$ $8.0^{\circ}$ square, positioned with its center at $7^{\circ}$ to the right of the fixation point, as shown in Figure 6 . Thus, it was centered over the simulated headings. The dot density remained the same as that for Experiment 1 , with an average of 36 dots visible at the beginning of the trial. The moving object was the same as in Experiment 1 and moved over a blank screen in the left half of the visual field. All other parameters were the same as in Experiment 1.

\section{Results}

The results of Experiment 3 were similar to those of Experiments 1 and 2, as diagrammed in Figure 7. The observers performed well on the heading task alone, with an average error of $0.4^{\circ}$. When performing both tasks, their performance level dropped slightly, with an average error of $0.9^{\circ}$ when attending the heading task and $1.2^{\circ}$ when attending the object task. Again, there was a slight increase of the central bias for the dual-task conditions over the single-task condition. An ANOVA showed a significant effect between the three conditions $[F(2,48)=$ $9.77, p<.01]$. Planned comparisons showed a significant difference between the single- and dual-task (attendheading) conditions $[F(1,48)=10.426, p<.05]$, but no significant difference between the two dual-task conditions $[F(1,48)=0.999, p>.3]$.

For the object task, as before, an ANOVA showed a significant effect of condition $[F(2,12)=58.10, p<.001]$. The observers responded with $97.8 \%$ correct for the object task alone and $93.3 \%$ correct when performing both tasks and attending the object task, with no significant difference between these two conditions, as shown by planned comparison $[F(1,12)=3.31, p>.12]$. Observer accuracy dropped to $72.8 \%$ correct when performing both tasks and attending the heading task, which was significantly less than the accuracy for the object task when the observers did both while attending the object $[F(1,12)=$ $68.75, p<.001]$. So, even when the stimuli cover similar spatial extents, we still see a difference in the effect of different allocations of attention on the heading task and the object task.

\section{EXPERIMENT 4 Short-Duration Task}

The results of Experiments 1-3 show that people's judgments of heading are only minimally affected when allocating their attention elsewhere. In these experiments, the duration of the trials was $0.4 \mathrm{sec}$. This duration was chosen to be long enough for observers to make accurate heading judgments (Crowell et al., 1990). This time allows a considerable amount of computation to occur within the visual system. Furthermore, the observers may be able to complete one task and then shift their attention to the other within this time. Therefore, the question arises as to what effects the observer's differential allocation of attentional resources might have for shorter presentation

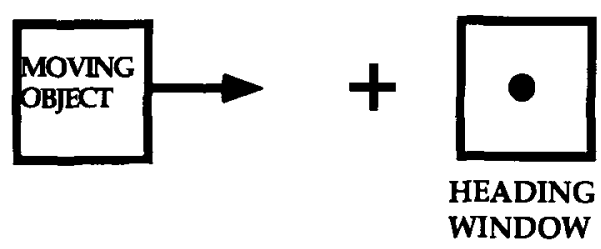

Figure 6. Schematic of image presented in Experiment 3. The square on the right indicates the window to which information about heading was confined. Dots moving outside this window disappeared from the screen. The rest of the screen was blank except for the moving object on the left side and the fixation cross in the center. 
A.

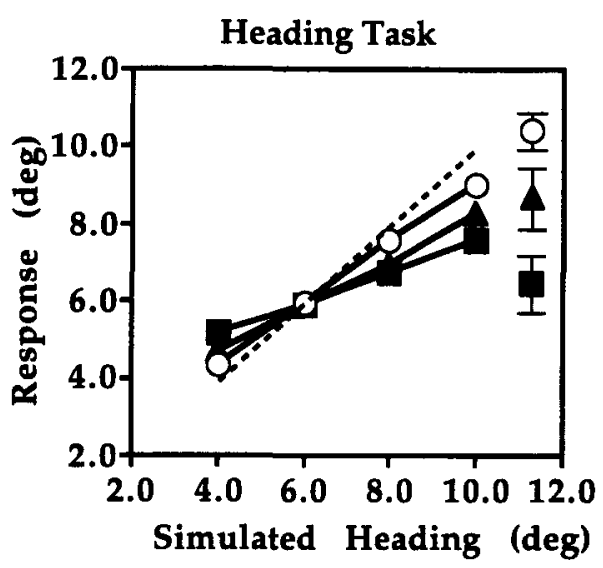

B.

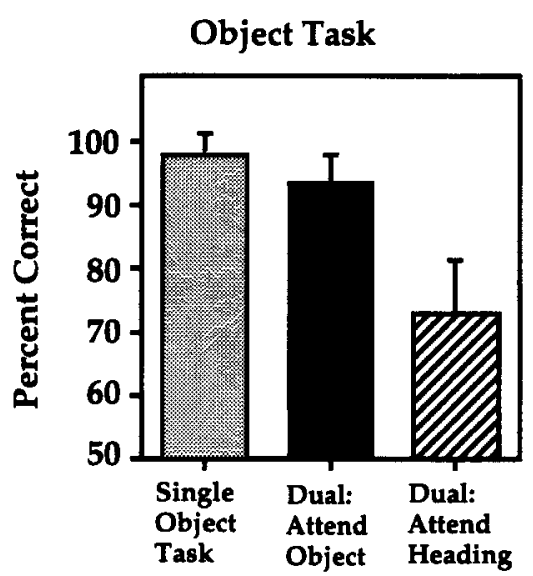

Figure 7. Results of Experiment 3, in which heading information was limited to a small square region. (A) Results of the heading task for the three heading conditions. (B) Results for the object task. All symbols are as described in Figure 3.

times. Shortening the presentation time could have the effect of making the task more difficult and thus increasing the computational load for processing the visual information. Alternatively, if observers are shifting their attention between the two tasks, the shorter time should not allow this. If either of these is the case, one might expect to see an effect of attentional allocation in this condition. We carried out Experiment 4 to examine the temporal properties of heading judgments for different allocations of attention.

\section{Method}

Experiment 4 was identical to Experiment 1 in all respects except for the trial duration, which was reduced to $0.2 \mathrm{sec}$.

\section{Results}

Figure 8 shows the results of Experiment 4 . At this short duration, the observers still performed the heading task well when doing that task alone, with an average error of $1.3^{\circ}$, although this was slightly worse than for the 0.4-sec duration. When performing both tasks and attending the heading, performance was similar, with an average heading error of $1.5^{\circ}$. However, when performing both tasks and attending the object, performance on the heading task deteriorated somewhat, with an average heading error of $2.1^{\circ}$. An ANOVA showed a nearly significant effect of condition $[F(2,48)=4.15, p<.07]$ and a significant interaction between the attention condition and the simulated heading $[F(6,48)=12.82, p<.01]$. This interaction can be seen as the marked decrease in the slope of the response curve for the dual-task attend-object condition. So, unlike the previous experiments, the heading task appeared to be affected by differential allocation of attention for this short-duration experiment.

Results for the object task were similar to those of Experiment 1, with a significant effect of condition $[F(2,12)=70.4, p<.0005]$. The observers responded with $91.3 \%$ correct for the object task alone and $88.5 \%$ correct when performing both tasks and attending the object. This difference was not significant $[F(1,12)=1.32$, $p>.2]$. When performing both tasks and attending head-

A.

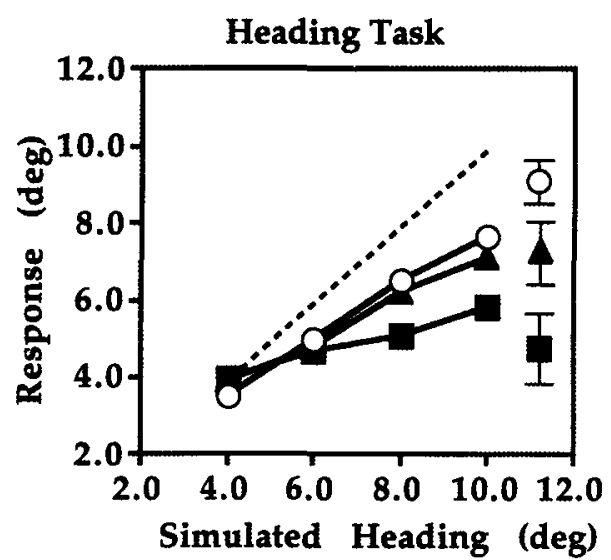

B.

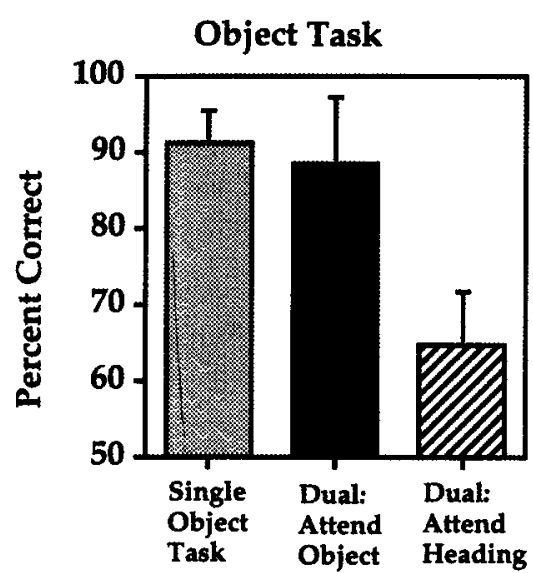

Figure 8. Results of Experiment 4, in which the duration of each trial was shortened to $0.2 \mathrm{sec}$. (A) Results of the heading task for the three heading conditions. (B) Results for the object task. All symbols are as described in Figure 3. 
ing, response accuracy dropped to $64.7 \%$ correct, significantly below the accuracy for the dual-task attendobject condition $[F(1,12)=93.2, p<.0005]$.

\section{EXPERIMENT 5 \\ Two-Alternative Forced Choice for Heading}

The question arises whether the difference in results between the heading and object tasks in Experiments 1-3 could be attributed to differences between the two response paradigms. One might argue that placing a cursor at the end of a trial (as in our heading task) is a more difficult task than responding with a buttonpress in a twoalternative forced choice (2AFC) task (as in our object task). If this were the case, then the heading task might draw more mental resources away from the object task than the reverse. In Experiment 5, we sought to make the observer responses for the two tasks as similar as possible by using the $2 \mathrm{AFC}$ task devised by Warren and Hannon $(1988,1990)$ to measure the accuracy of the heading judgments.

Another important factor in measuring the effects of attentional allocation in dual-task experiments is the relative difficulty of performing the two tasks. In Experiment 4 , the heading task was somewhat more difficult than that in Experiments 1-3 because of the shorter duration. The results suggest that this increase in difficulty begins to affect observers' accuracy in judging heading when attentional resources are allocated elsewhere. To further examine this, we varied the difficulty of both the heading and the object tasks in Experiment 5.

\section{Method}

The stimuli in Experiment 5 were identical to those in Experiment 1 , except that a vertical line appeared at the end of each trial. For each trial, the horizontal heading was chosen at random between the limits of $4^{\circ}$ and $10^{\circ}$ to the right of the fixation point, and the vertical heading was chosen between the limits of $\pm 2^{\circ}$ from the horizontal midline. At the end of each trial, a vertical target line was placed $1^{\circ}, 2^{\circ}$, or $3^{\circ}$ to the left or right of the simulated heading, with the $1^{\circ}$ target offset being the most difficult condition and the $3^{\circ}$ offset the easiest. In the experimental runs that required a heading judgment, the observers indicated with a buttonpress whether their heading was to the left or right of the vertical line. To vary the difficulty of the object task, we varied the magnitude of the component of motion in depth with respect to the observer. In separate runs of the experiment, this component was 1, 1.5 (as in Experiments 1-4), or 2.0 times the magnitude of the horizontal component. A fifth attentional allocation condition was added in which the observers were instructed to pay equal attention to both the object and the heading tasks. This was added for the purpose of constructing the performance operating characteristics (POCs) described below. In each run, each of the 12 conditions ( 3 target offsets $\times 2$ target line directions $\times 2$ object conditions) was repeated 10 times for a total of 120 trials. Two of the observers from Experiment 1 and 3 new naive observers participated in this experiment. Two of these had not previously participated in psychophysical experiments.

\section{Results}

The six graphs in Figure 9 show the results of Experiment 5. Each bar shows the percent of correct responses averaged over the 5 observers and the 120 trials in each run of the experiment. The graphs in Figures 9A, 9C, and $9 \mathrm{E}$ show the results for the heading task in the hard, medium, and easy object task conditions. Each graph shows the result for three target offsets, with the $1^{\circ}$ offset being the most difficult and the $3^{\circ}$ offset being the easiest. The gray bars show the percent of correct responses for the heading task alone. The black, checked, and striped bars show the results for the dual-task attendheading, attend-both, and attend-object conditions, respectively. Examination of the data reveals a small decrease in accuracy between the single-task (gray bars) and dual-task (black bars) conditions in nearly all the levels of difficulty except for the easiest conditions for both the heading and object task (Figure 9E, $3^{\circ}$ offset). For the majority of conditions, there was little difference in accuracy between the dual-task conditions, whether the observer attended the heading task (black bars), both tasks (checked bars), or the object task (striped bars). A threeway ANOVA (repeated measures, using the GreenhouseGeisser correction), with heading-task difficulty, objecttask difficulty, and attention condition as factors, showed that only the difficulty of the heading task had a significant effect $[F(2,144)=45.43, p<.005]$.

Results for the object task are diagrammed in Figures 9B, 9D, and 9F for the difficult, medium, and easy object task conditions. The gray bars show results for the object task alone. The black, checked, and striped bars show the results for the dual-task attend-object, attend-both, and attend-heading conditions, respectively. The data show that there was little difference between the single-task and the dual-task attend-object conditions in any of the experiments. In contrast to the heading results, there was a consistent decrease in the percent of correct responses, with the dual-task attend-object condition (black bars) giving the most accurate responses, the dual-task attend-both condition (checked bars) giving an intermediate level, and the dual-task attend-heading condition (striped bars) giving the worst accuracy. A three-way ANOVA showed significant effects for object difficulty $[F(2,144)=4.77$, $p<.05]$ and attention $[F(3,144)=30.75, p<.005]$. Planned means comparisons showed a significant difference between the dual-task condition in which the observers attended the object and the dual-task condition in which they attended heading $[F(1,144)=69.39, p<.0001]$.

Thus, the basic result of Experiments 1-3 holds for all of the combinations of difficulty shown here. The object task shows a significant decrease in accuracy when attention is allocated away from the task, whereas the heading task shows remarkably little effect of different attentional allocations.

\section{DISCUSSION}

With these experiments, we tested the effect on heading and object-motion judgments of asking observers to attend one task or the other in a dual-task experiment. The results suggest a small effect of going from the single- 


\section{Difficult Object Condition}
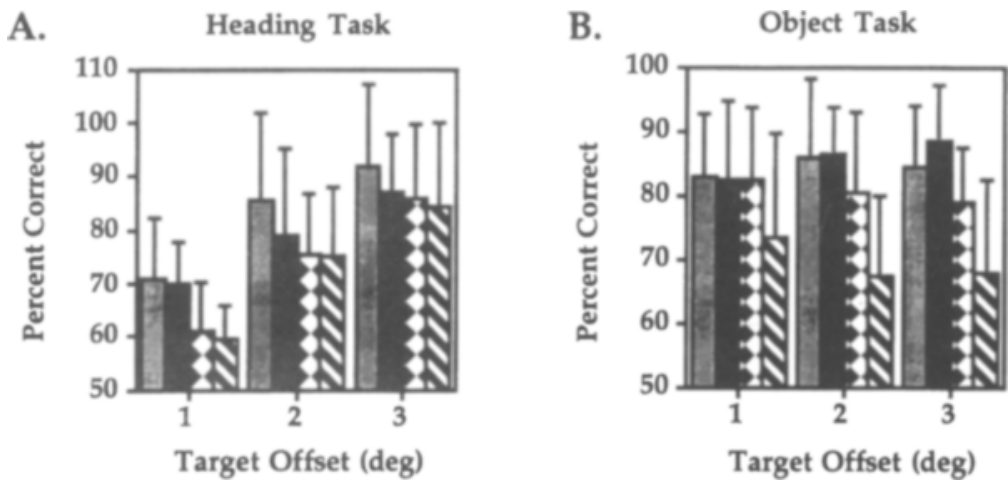

\section{Medium Object Condition}
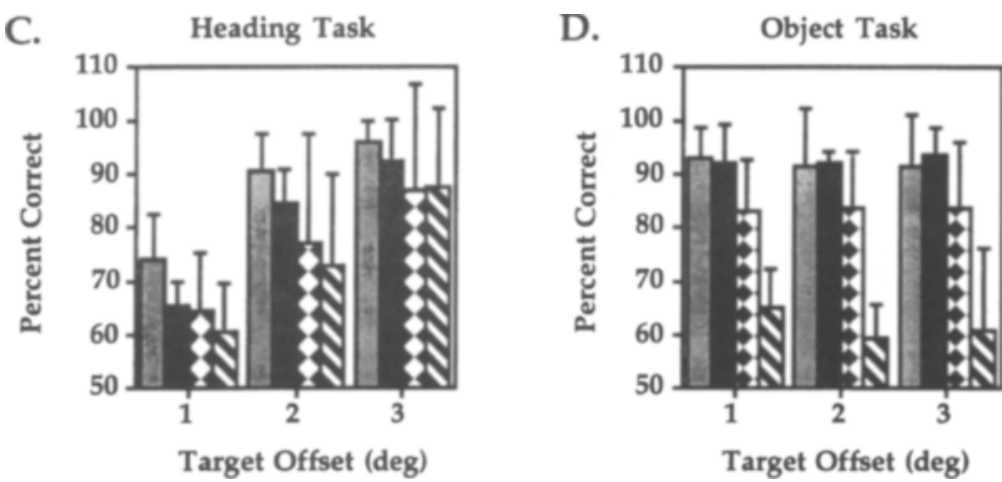

Easy Object Condition
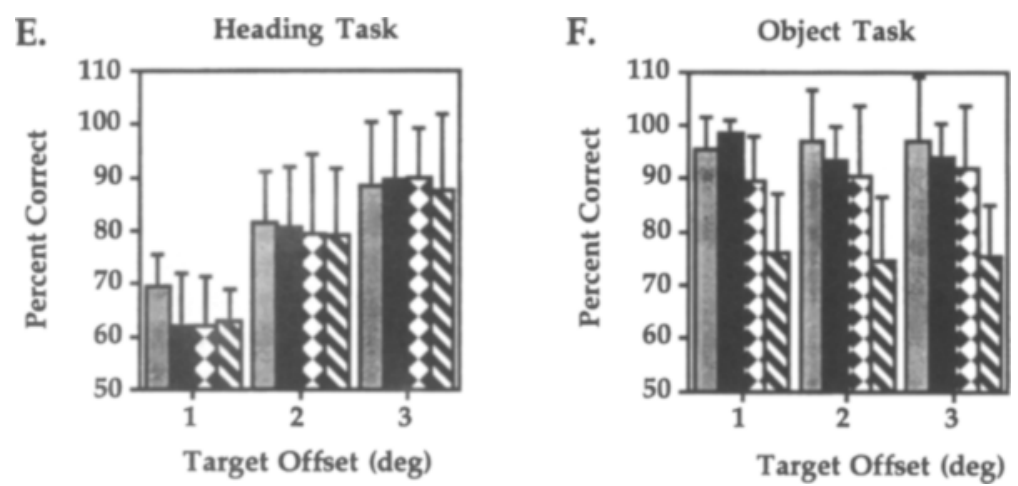

Figure 9. Results of Experiment 5 using a two-alternative forced choice task to measure heading discrimination while varying task difficulty. Panels $A$ and B: Results for the heading and object tasks, respectively, for the hardest object condition (depth component 1.0 times the horizontal component). Panels $C$ and $D$ : Results for the heading and object tasks, respectively, for the middle object condition (depth component 1.5 times the horizontal component). Panels $E$ and $F$ : Results for the heading and object tasks, respectively, for the easiest object condition (depth component 2.0 times the horizontal component). Each graph shows the results for target offsets of $1^{\circ}, 2^{\circ}$, and $3^{\circ}$ for the heading. The shaded bars show the percent of correct responses, averaged over 5 observers, when the observers performed a single task alone (heading task for Panels $A, C$, and E; object task for Panels B, D, and F). The black bar shows the results when the observer performed both tasks, but attended the task being graphed (attendheading task for Panels $A, C$, and E; attend-object task for Panels $B, D$, and F). The checked bars show the results for the observers attending both tasks equally. The hatched bars show the percent correct when the observers performed both tasks, but attended the task not being graphed (attend-object task for Panels $\mathrm{A}, \mathrm{C}$, and E; attend-heading task for Panels B, D, and F). 
task condition to the dual-task conditions, particularly in the heading task. More importantly, in the dual-task conditions, there was a large effect of shifting attention for the object task, but very little effect for the heading task.

Comparison of the heading results of the single-task condition with those of the dual-task condition in which the observers attended heading, reveals a small, consistent decline in heading accuracy when the second task is added. The same is true for the object task. These decreases were statistically significant in some of the conditions tested (Experiments 2 and 3 ). The consistency of the decline across all five experiments suggests that it is meaningful. An ANOVA of the combined data from Experiments 1-4 showed a significant difference in heading results between the single-task condition and the dualtask attend-heading condition $[F(1,160)=4.99, p<$ .03]. A similar significant result was found for the object task when comparing the combined results of the singletask with the dual-task attend-object condition $[F(1,37)=$ $6.66, p<.05]$. Thus, as one might expect, there was a small effect on both tasks of adding a second task to the experiment.

A more interesting observation comes from comparing the results of the different attentional states for the dual-task conditions. The results of our experiments show that, for these dual-task conditions, heading judgments remain accurate even when the observer pays attention to the object task. In contrast, the observers could not judge the 3-D direction of object motion when not paying attention to this task. One way to visualize this difference clearly is to generate graphs of the attention operating characteristic (AOC), also known as the $P O C$ curves (Norman \& Bobrow, 1975; Sperling \& Dosher, 1986; Wickens, 1992). These graphs plot the percent correct for the heading judgments against the percent correct for the object judgments as the observers change their attentional allocation. Figure 10 shows these graphs for the data from Experiment 5. Clearly, in the dual-task condition, the curves are nearly horizontal for almost all the conditions tested. This means that the accuracy of heading judgments is not affected much by different allocations of the observer's attention, but that the accuracy in the object task is strongly affected. In the terms used by Norman and Bobrow (1975), the heading task is data limited, meaning that the accuracies do not improve with increased attentional resources. On the other hand, the object task is resource limited, with the accuracy depending strongly on the attentional resources allocated to the task. The one exception to this analysis is for the case in which the most difficult heading task is combined with the most difficult object task. In this case, shown in Figure $10 \mathrm{C}$, when both tasks are near threshold (around $70 \%$ correct), we see a more symmetrical effect of differential allocation of attention. The form of this graph is generated by a single data point, for the case in which observers are performing both tasks while attending heading. In this condition, the observers performed slightly better than expected on both tasks. It would be a mistake to draw any strong conclusions on the basis of a single data point,

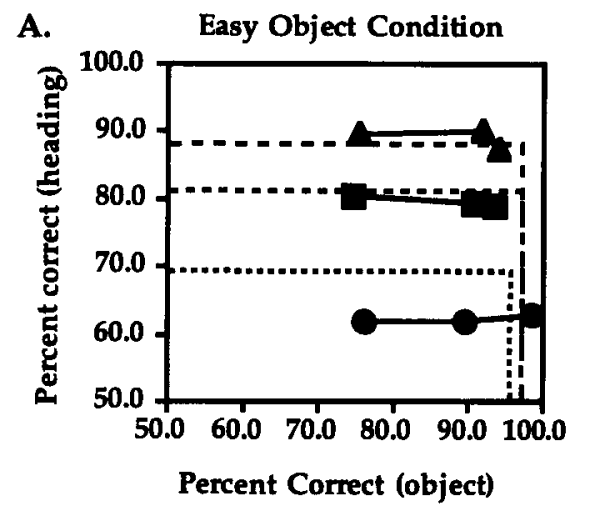

B. Medium Object Condition

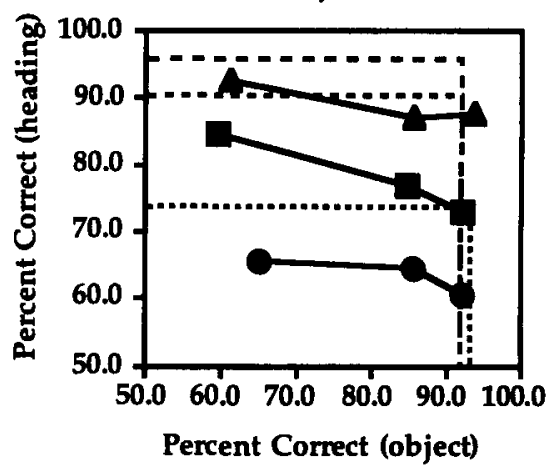

C. Difficult Object Condition

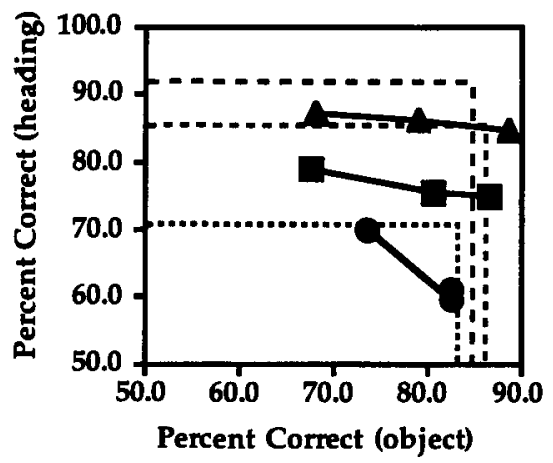

Figure 10. Plots of attention operating characteristic (AOC) curves for Experiment 5. Each graph plots the percent of correct responses for the heading task against the percent correct for the object task for a given experimental condition. The three data points for each curve represent conditions in which the observers attended heading, attended both tasks equally, and attended the object task. Triangles, squares, and circles show results for the $3^{\circ}$, $2^{\circ}$, and $1^{\circ}$ target offsets, respectively. Dashed lines show the singletask results for each of the target offsets (long dashes, $3^{\circ}$ offset; short dashes, $1^{\circ}$ offset). Panels $A, B$, and C: The $A O C$ curves for the easy, medium, and difficult object conditions (motion in depth 2.0, 1.5 , and 1.0 times the horizontal motion component), respectively.

but the behavior of observers in these tasks when they are near threshold deserves further investigation.

The fact that differential allocation of attention does not greatly affect heading judgments for the dual-task conditions is unexpected. Land and Lee (1994) showed 
that drivers frequently center their gaze in the direction they are steering, which suggests that it is important to look where one is going for successful navigation. However, they also point out that the steering task allows for considerable time-sharing with other tasks, such as viewing the scenery, since the drivers' gaze frequently veered from the road, especially during straight portions of the route. Previous experiments with airline pilots in flight simulators have shown that increase in workload has a decided effect on the pilot's ability to maintain an accurate flight path (see, e.g., Raby \& Wickens, 1994). It is difficult to compare the results of those experiments to ours, because there are so many factors in the tasks the pilots must perform in the flight simulator: judgments of heading, accurate control of the plane to maintain a desired heading, conversations with air traffic control, filling out written reports, and so on. Pilots almost certainly are not always looking at the visual stimulus to gauge their heading as they perform these different tasks. Therefore, one cannot say with certainty which factors affect the pilot's performance, or which part of the performance is affected (e.g., the lack of ability to keep on an accurate flight path could result from inaccurate judgments of heading or from inability to perform the motor task well when attention is diverted elsewhere). Our experiments examined one small piece of this puzzle. Because the observers fixated a central cross, the visual stimulus was the same for all the conditions tested within each experiment, and the observers were not required to maintain a particular course but were asked simply to report their perceived heading. Furthermore, we measured heading judgments only for observer motion in a straight line, so it is possible that the curved trajectories used in Raby and Wickens and Land and Lee may require more attention for accurate perception. Our results suggest that, assuming the observers are looking roughly in the direction they are traveling, the accuracy of their heading judgments for motion in a straight line is minimally affected by different allocations of attention. The fact that we saw a small, but consistent, effect on heading judgments of changing between single- and dual-task experiments suggests that adding extra motor tasks may affect the ability to perceive or report headings accurately. If so, one might expect the allocation of attention to affect the ability of an observer to maintain a given trajectory, as seen in the Raby and Wickens study. This remains to be tested.

\section{Differences Between Heading and Object Tasks}

The lack of symmetry in the heading and object task results is somewhat surprising because, in many respects, the tasks are rather similar. They both require the observer to make a judgment regarding his or her own motion relative to an object or scene. Thus, we expected that if an observer's allocation of attention affected one task, it would likely affect the other in the same way. The fact that differential allocation of attention affected the object task more dramatically than the heading task led us to examine some of the differences between the two stimuli and tasks. With Experiments 2-5, we were able to rule out the spatial position of information about the task, the amount of information present in the stimulus, the type of response (2AFC vs. cursor placement), and the difficulty of the task as critical differences responsible for the differing results.

We do not know why attention affected the two tasks differentially; however, several possibilities remain. One possibility is the difference in the overall direction of motion of the scene and the object relative to the observer. The simulated motion of the observer is nearly straight toward the scene, whereas the simulated motion of the object is oblique. Crowell and Banks (1993) pointed out that observer thresholds for discriminating headings are much higher for oblique motion than for more forward motion. One might conclude that the moving-object task is more difficult than the heading task because of the oblique motion of the object relative to the observer, which leads to the deterioration of accuracy on that task when the observer shifts his or her attention away. This seems unlikely for several reasons. First, the angular difference between the horizontal object motion and the motion of the object as it approached the observer was large, at $63^{\circ}$ in Experiments 1-4. This is over twice the threshold for detecting a difference in heading direction at oblique angles reported by Crowell and Banks (1993). In our experiments, observer accuracy on the object task when performed alone was very high-a fact that supports our argument that the two motions were well above threshold for discrimination. This fact weighs against the argument that the moving-object task was more difficult than the heading task in Experiments 1-4. Furthermore, in Experiment 5, we varied the difficulty of both the object task and the heading task. With the exception of a single data point, for the case in which both tasks were performed near threshold, the object task was greatly affected when the observers shifted their attention away from the task, whereas the heading task was affected much less. Thus, the relative difficulty of the two tasks cannot account for our results. It is still possible that oblique directions of motion are more susceptible to shifts of attention than is forward motion. This possibility remains to be tested in future experiments.

Another difference between the moving-object task and the heading task is that the object borders changed position over time, whereas the heading window stayed in one place. Perhaps this change in position of the object borders caused the visual system to process the information differently than the information from the heading window. One problem often occurring for computational models of human heading is how to determine which image motion vectors are associated with moving objects and which are associated with the stationary scene. 
Perhaps this change in position acts as a cue to the visual system that the motion belongs to a moving object. This idea remains to be tested.

\section{Temporal Properties}

In Experiment 4, we tested the effects of the observers' focus of attention using a very short presentation time for each trial. We found that, in this condition, the observers' heading judgments did deteriorate somewhat when their attention shifted away from that task, as shown by the decrease in the slope of the response curve for the heading task when the observers attended the object task. There are several possible reasons for the differences in results between this experiment and the other experiments. One possibility is that the observers could make a rapid judgment of the 3-D object motion and then shift their attention to the heading task in Experiments 1-3. In Experiment 4, the decreased amount of time did not allow this. One argument against this interpretation is that if observers accomplished their accurate results for heading by shifting their attention, one would expect them to do the same for the object task. Thus, one would also expect good results for the object task when attending heading. Another argument against this interpretation is that the observers were instructed to attend to the heading or the object throughout the trial. The observers reported that they followed this instruction and did not consciously shift their attention.

A second possible explanation for the deterioration in heading accuracy in Experiment 4 is that the heading task is harder for the shorter duration stimulus, and, therefore, it is more sensitive to the differential allocation of attention. We did not see this effect when the difficulty of the heading task was varied in Experiment 5 by changing the position of the target line with respect to the simulated heading; however, it is conceivable that manipulating the difficulty by different methods could lead to varied results.

Another possible reason for the results in Experiment 4 is that accurate heading judgments could require more temporal integration time if observers are not actively attending to the heading task. In this scenario, a certain level of computational resources would be available to the heading task at all times, irrespective of the focus of attention of the observer. Focusing one's attention on the heading task would be akin to allocating more computational resources to that particular task. The extra resources may allow more rapid computation of heading than the baseline mechanisms. Thus, for very short durations, the focused attention state would allow accurate heading computation, whereas the baseline mechanisms would compute a coarser heading estimate. As the computation time is increased, the baseline mechanisms could further refine the initial heading computations to obtain a more accurate heading judgment.

Reaction time studies might be one way to address some of these issues. It is possible that, in Experiments $1,2,3$, and 5 , the observers were able to maintain a high accuracy in heading judgments by allowing more processing time when they attended the object task. A longer reaction time would allow more processing resources to be devoted to the heading computation. The results of Experiment 4 could be a reflection of the need for longer processing time for maintaining accurate heading judgments. Thus, it is possible that reaction time studies might show some effect of attentional shifts in the heading task that is not seen with accuracy studies alone. However, even if this were so, it would not explain the differing results between the heading and object tasks. If the observers did show an increased reaction time when performing the two tasks together, it is unclear why this increased processing time would increase heading accuracy but not increase accuracy in the object task.

We cannot currently distinguish between these possible explanations of the results of Experiment 4: rapid attentional shifts, differences in difficulty, or temporal integration time. The result is intriguing, however, and further investigation could lead to valuable insights into the mechanisms underlying heading judgments.

\section{Implications for Computational Models}

As discussed in the introduction, several models are consistent with the idea that heading computations are done in parallel across the visual field without the need for focused attention for the most accurate judgments. Two types of models that would be consistent with this are the template models of Perrone and Stone (1994) and Warren and Saunders (1995) or the vector subtraction model of Hildreth (1992). All of these models use all the information available in the visual scene to estimate heading. Thus, there is no need to focus additional computational resources on one particular region of the flow field (e.g., the region around the FOE). The idea that heading is processed globally is also consistent with the results of Dyre and Andersen (1997), who showed that asymmetries in the flow field can affect heading judgments. The results of Experiment 4, however, suggest that one should be careful in concluding this. The fact that accurate heading judgments may require more time when attention is not focused on the heading task suggests that the most accurate heading judgments may involve sequential processing. The model suggested by Royden and Hildreth (1996), in which an initial rough estimate is subsequently finetuned using information near the estimated FOE, would be consistent with this result.

\section{Relation to Physiology}

Because all responses in these experiments ultimately depend on the underlying neuronal mechanisms, it is instructive to consider the underlying physiology of the visual areas thought to be involved in computing heading and object motion. It seems likely that information concerning both heading and object motion is analyzed in the medial superior temporal area (MST) of visual cortex. Heading is thought to be processed in the dorsal region (MSTd), because cells in that area have large re- 
ceptive fields and prefer stimuli that are similar to the flow fields generated by relative motion between an observer and an object or scene, such as expanding, contracting, or rotating stimuli, or stimuli that move laterally (Duffy \& Wurtz, 1991a, 1991b; Graziano, Andersen, \& Snowden, 1994; Saito et al., 1986; Tanaka, Fukada, \& Saito, 1989; Tanaka \& Saito, 1989). This receptive field organization is consistent with the idea that processing of heading occurs in parallel across the visual field.

In contrast to MSTd, neurons in the lateral part of MST (MSTl) are thought to process object motion because they have smaller receptive fields than those in MSTd and respond well to small spots of light (Komatsu \& Wurtz, 1988; Newsome, Wurtz, \& Komatsu, 1988). Perhaps these neurons are more sensitive to the focus of the observer's attention, with an enhancement of response when the observer attends to the location corresponding to the cell's receptive field. This would be consistent with our finding that the allocation of attention does affect the observer's ability to judge object motion.

Treue and Maunsell (1996) recently reported that the response of directionally selective cells in superior temporal sulcus (STS), the region in which MT and MST are located, is affected by shifts in attention. They showed that when the two spots were both located within a cell's receptive field with one moving in the cell's preferred direction of motion and the other moving in the antipreferred direction, the cell responded more strongly when the monkey attended the spot that was moving in the preferred direction of motion. The fact that a shift of attention affects the firing of neurons in STS is consistent with the effect of attention that we see in our moving-object task. Thus, if the neurons observed by Treue and Maunsell were involved in computing object motion, these two results would be compatible. Unfortunately, it is unclear in their initial report whether there is a differential effect between the responses of cells in MSTd and MSTI. Because our experiments show that heading estimates are less affected by attentional focus than are judgments of object motion, we would predict that the responses of cells in MSTd are less affected by the attentional state of the monkey than are responses of cells in MSTl.

\section{Summary}

We have shown that when performing both a heading task and an object-motion task people's heading judgments are minimally affected by shifting attention to the object task, unless the duration of the stimulus is very short. For $400-\mathrm{msec}$ trials, there was little difference in the observers' heading responses whether they paid attention to the heading task or to a 3-D object-motion task. In contrast, shifting attention away from the object task dramatically decreased accuracy in judging the direction of 3-D object motion. This suggests that judgments of heading are computed globally throughout the visual field, whereas judgments of object motion require more focused attention. Future experiments will help define how the visual system differentiates heading information from moving-object information and how this leads to the differing effects of attention.

\section{REFERENCES}

Bruss, A. R., \& HoRn, B. K. P. (1983). Passive navigation. Computer Vision, Graphics, \& Image Processing, 21, 3-20.

Crowell, J. A., \& Banks, M. S. (1993). Perceiving heading with different retinal regions and types of optic flow. Perception \& Psychophysics, 53, 325-337.

Crowell, J. A., \& Banks, M. S. (1996). Ideal observer for heading judgments. Vision Research, 36, 471-490.

Crowell, J. A., Royden, C. S., Banks, M. S., Swenson, K. H., \& SEKULER, A. B. (1990). Optic flow and heading judgments. Investigative Ophthalmology \& Visual Science, 31(Suppl.), 522.

Cutting, J. E., SPringer, K., Braren, P. A., \& Johnson, S. H. (1992). Wayfinding on foot from information in retinal, not optical, flow. Journal of Experimental Psychology: General, 121, 41-72.

Cutting, J. E., Vishton, P. M., \& Braren, P. A. (1995). How we avoid collisions with stationary and moving obstacles. Psychological Review, 102, 627-651.

DUFFY, C. J., \& WURTZ, R. H. (1991a). Sensitivity of MST neurons to optic flow stimuli: I. A continuum of response selectivity to large-field stimuli. Journal of Neurophysiology, 65, 1329-1345.

DUFFY, C. J., \& WURTZ, R. H. (1991b). Sensitivity of MST neurons to optic flow stimuli: II. Mechanisms of response selectivity revealed by small-field stimuli. Journal of Neurophysiology, 65, 1346-1359.

DYRE, B. P., \& ANDERSEN, G. J. (1997). Image velocity magnitudes and perception of heading. Journal of Experimental Psychology: Human Perception \& Performance, 23, 546-565.

Geisser, S., \& Greenhouse, S. W. (1958). An extension of Box's results on the use of the $F$ distribution in multivariate analysis. Annals of Mathematical Statistics, 29, 885-891.

Graziano, M. S. A., Andersen, R. A., \& Snowden, R. J. (1994). Tuning of MST neurons to spiral motions. Journal of Neuroscience, 14, 54-67.

Hatsopoulos, N. G., \& WARREN, W. H. (1991). Visual navigation with a neural network. Neural Networks, 4, 303-317.

HeEgER, D. J., \& JEPSON, A. D. (1992). Subspace methods for recovering rigid motion: I. Algorithm \& implementation. International Journal of Computer Vision, 7, 95-117.

HILDRETH, E. C. (1992). Recovering heading for visually-guided navigation. Vision Research, 32, 1177-1192.

Hildreth, E. C., \& RoYden, C. S. (1996). Computing observer motion from optical flow. In T. Watanabe (Ed.), High-level motion processing: Computational, neurobiological, and psychophysical perspectives (pp. 269-293). Cambridge, MA: MIT Press.

Johnston, I. R., White, G. R., \& Cumming, R. W. (1973). The role of optical expansion patterns in locomotor control. American Journal of Psychology, 86, 311-324.

Komatsu, H., \& WurTz, R. H. (1988). Relation of cortical areas MT and MST to pursuit eye movements: I. Localization and visual properties of neurons. Journal of Neurophysiology, 60, 580-603.

LAND, M., \& LEE, D. N. (1994). Where we look when we steer. Nature, $369,742-744$.

LLEWELLYN, K. R. (1971). Visual guidance of locomotion. Journal of Experimental Psychology, 91, 245-261.

Longuet-Higgins, H. C., \& PRAZdNY, K. (1980). The interpretation of a moving retinal image. Proceedings of the Royal Society of London $B, 208,385-397$.

Newsome, W. T., Wurtz, R. H., \& Komatsu, H. (1988). Relation of cortical areas MT and MST to pursuit eye movements: II. Differentiation of retinal from extraretinal inputs. Journal of Neurophysiology, 60, 604-620.

Norman, D. A., \& Bobrow, D. G. (1975). On data-limited and resourcelimited processes. Cognitive Psychology, 7, 44-64.

Perrone, J. A., \& Stone, L. S. (1994). A model of self-motion estimation within primate extrastriate visual cortex. Vision Research, $\mathbf{3 4}$ 2917-2938. 
Raby, M., \& WiCKENS, C. D. (1994). Strategic workload management and decision biases in aviation. International Journal of Aviation Psychology, 4, 211-240.

Rieger, J. H., \& Lawton, D. T. (1985). Processing differential image motion. Journal of the Optical Society of America A, 2, 354-360.

RIEGER, J. H., \& TOET, L. (1985). Human visual navigation in the presence of 3D rotations. Biological Cybernetics, 52, 377-381.

Royden, C. S., Banks, M. S., \& Crowell, J. A. (1992). The perception of heading during eye movements. Nature, 360, 583.585.

Royden, C. S., Crowell, J. A., \& Banks, M. S. (1994). Estimating heading during eye movements. Vision Research, 34, 3197-3214.

RoYden, C. S., \& HildreTH, E. C. (1996). Human heading judgments in the presence of moving objects. Perception \& Psychophysics, 58, 836-856.

Saito, H., Yukie, M., Tanaka, K., Hikosaka, K., Fukada, Y., \& IWAI, E. (1986). Integration of direction signals of image motion in the superior temporal sulcus of the macaque monkey. Journal of Neuroscience, 6, 145-157.

SPERLING, G., \& Dosher, B. A. (1986). Strategy and optimization in human information processing. In K. R. Boff, L. Kaufman, \& J. P. Thomas (Eds.), Handbook of perception and human performance: I. Sensory processes and perception (pp. 2-I to 2-65). New York: Plenum.

Tanaka, K., Fukada, Y., \& SaITo, H. (1989). Underlying mechanisms of the response specificity of expansion/contraction and rotation cells in the dorsal part of the medial superior temporal area of the macaque monkey. Journal of Neurophysiology, 62, 642-656.
TANAKA, K., \& SaITo, H. (1989). Analysis of motion in the visual field by direction, expansion/contraction, and rotation cells clustered in the dorsal part of the medial superior temporal area of the macaque monkey. Journal of Neurophysiology, 62, 626-641.

Thompson, W. T., Lechleider, P., \& STUCK, E. R. (1993). Detecting moving objects using the rigidity constraint. IEEE Transactions on Pattern Analysis \& Machine Intelligence, PAMI-15, 162-166.

Thompson, W. T., \& PonG, T. C. (1990). Detecting moving objects. International Journal of Computer Vision, 4, 39-57.

Treue, S., \& MAUNSELL, J. H. R. (1996). Attentional modulation of visual motion processing in cortical areas MT and MST. Nature, 382, 539.

VAN DEN BERG, A. V. (1992). Robustness of perception of heading from optic flow. Vision Research, 32, 1285-1296.

WARREN, W. H., \& HANNON, D. J. (1988). Direction of self-motion is perceived from optical flow. Nature, 336, 162-163.

WARREN, W. H., \& HANNON, D. J. (1990). Eye movements and optical flow. Journal of the Optical Society of America A, 7, 160-169.

WARren, W. H., \& KurTZ, K. J. (1992). The role of central and peripheral vision in perceiving the direction of self-motion. Perception \& Psychophysics, 51, 443-454.

Warren, W. H., \& Saunders, J. A. (1995). Perceiving heading in the presence of moving objects. Perception, 24, 315-331.

WICKENS, C. D. (1992). Engineering psychology and human performance (2nd ed.). New York: Harper Collins.

(Manuscript received March 15, 1996; revision accepted for publication December 26, 1997.) 